\title{
IoT system for remote monitoring of the mangrove forests of Sundarbans
}

\section{System IoT do zdalnego monitorowania lasów namorzynowych Sundarbans}

\author{
Asif Rahman Rumee* \\ Department of Computer Science and Engineering, Jashore University of Science and Technology, Jashore-7408, \\ Bangladesh
}

\begin{abstract}
In-situ monitoring of mangrove forests is expensive, cumbersome, time consuming and error-prone, hence remote approaches are being used widely nowadays. Remote sensing using satellites, UAVs and other devices is incapable of collecting many important types of data required for processing, therefore a prototype of an IoT device is designed and built for monitoring environmental parameters of the largest mangrove forests in the world, the Sundarbans in Bangladesh. The prototype is tested for a few hours in a simulated environment where the readings are updated every 2 seconds and alert notifications are received if an emergency event occurs. The simulation results prove the effectiveness of the proposed device and the feasibility of it for low cost remote monitoring of the mangrove forests.
\end{abstract}

Keywords: remote monitoring; IoT; mangrove forests; Sundarbans

*Corresponding author

Email address: arrumee@gmail.com (A. R. Rumee)

CPublished under Creative Common License (CC BY-SA v4.0)

\section{Introduction}

Generally remote sensing using satellites, aeroplanes and unmanned aerial vehicles etc. [1-2] are being used nowadays to capture imagery and related data for mapping and monitoring mangrove forests in different parts of the world. Before the appearance of the remote sensing in-situ monitoring [3] approach was used involving a huge amount of human resources to collect the required data from widespread adverse areas. On-site monitoring process was tedious and monotonous due to the repetitive nature of the tasks belonged to it. It was error-prone as well due to human intervention. This approach also used to take a lot of time to perform the tasks and it was a very high cost process. On the contrary the remote sensing approaches used currently are easy to conduct, more accurate and less expensive compared to in-situ approaches [4]. But this approach has some limitations to such as unable to collect all required data [5]. Therefore, people are looking for some other techniques to overcome these issues. With the advancement of embedded systems, sensors, wireless networks IoT systems have become a very common mechanism for remote monitoring and tracking of many objects and parameters in agriculture, industries, healthcare, transportation, supply-chain management sectors and so on. Some IoT devices can measure the concentration of different gases such as $\mathrm{CO}_{2}, \mathrm{CO}, \mathrm{NO}_{2}$ present in the air as well as temperature, humidity and send it to the distant servers [6]. Sound level and noise in smart cities can be measured using some autonomous devices as well [7]. Besides air and sound quality almost all kinds of environmental variables are being measured and analysed using IoT based systems. Corn, rice, vegetables, fruits are cultivated with the help of smart IoT devices. These devices monitor the water level, soil moisture, soil nutrients etc. and then according provide required amount of water, fertilizer and necessary things [8-9].

The Sundarbans are the largest mangrove forests in the world, located at the southern region of Bangladesh on shore of Bay of Bengal and on the estuary of Ganga, Brahmutra, Meghna along with the west Bengal of India. The total area of it is about 10,000 square kilometres where 6,000 square kilometres belong to Bangladesh. It was declared one of the World Heritage Sites by UNESCO in 1997. It alone contributes $40 \%$ to the total forest land of Bangladesh. It plays a vital role for maintaining ecological and environmental balance in Bangladesh. Therefore, often she is called the lunge of our country. She protects us from natural disasters like cyclones, storms, floods etc. many times in every year. It is important for our country not only for environmental aspects but also for economic aspects. The ecosystem of the Sundarbans is degrading day by day which have become a serious threat to Bangladesh as well as the whole world. The average yearly revenue earned from provisioning services of the forest e.g. timber, fuel wood, fishes, thatching materials, honey and waxes, was 744,000 US dollars. The revenue from cultural services i.e. tourism was on an average 42,000 US dollars per year during the fiscal years 2001-2002 to 2009-2010 [10]. The economic contributions of the forest to lowincome, middle-income and high-income population living adjacent to it were $74 \%, 48 \%$ and $74 \%$ of per capita annual income respectively [11]. Actually it is not possible to express the importance of the Sundarbans in terms of both ecological and economic perspectives by words. It has blessed the people in many ways but in response the people are causing harms to 
her gradually. The water in the rivers of the forest is being contaminated by oil spillage by ships, pesticides used in near fields and farms. Industrial factories near the forest such coal power plants are continuously polluting the air quality by emitting toxic gases e.g. $\mathrm{SO}_{2}$, $\mathrm{NO}_{2}$ etc. Therefore, an effective monitoring system is needed to correctly measure the pollution of the environment of the forest in order to save it from degradation.

In this paper a prototype of an IoT device for low cost remote monitoring of the mangrove forests of Sundarbans is studied. The different functionalities of the device such as air, water, soil and sound quality measurement are checked as well. This research focuses on measuring the efficiency of the prototype as well as the feasibility of it for remote monitoring of the mangrove forests in a lower cost.

\section{Materials and Methodology}

\subsection{Materials}

A networking and IoT simulation tool named cisco packet tracer [12] is used to build the prototype as well as to make the test environment. From many different kinds of sensors available in the tool 8 types of sensors are used in building the prototype. One microcontroller board is used with a wireless module attached to it. In the remote station one single board computer is used as a server (see Figure 1). A list of these components is described below briefly.

- 1 MCU-PT microcontroller. Equivalent real device available in the market is Arduino Uno which costs 16 US dollars [13] and Wireless module SIM808 which costs 18 US dollars [14].

- 1 Humidity Sensor that provides analog output in range 0 to 255 , representing 0 to $100 \%$ humidity in the air whose equivalent real device is DHT11 sensor.

- 1 Smoke or Gas Sensor which gives analog output in range 0 to 1023 , representing 0 to $100 \%$ smoke percentage in the air that is similar to real device MQ-2 Gas Smoke sensor.

- 1 Water Sensor which gives analog output from 0 to 1023 representing 0 to $20 \mathrm{~cm}$.

- 1 Sound Sensor which gives analog output as sound level in decibels.

- 1 Temperature Sensor which gives analog output in range 0 to 255 , representing $-100 \mathrm{C}$ to $100 \mathrm{C}$ temperature ranges that is equivalent to real sensor DS18B20.

- 1 Water Detector that detects presence water seems to be a rainfall detector.

- 1 Wind or Vibration Sensor that detects wind in the environment.

- 1 Fire or Flame Sensor that detects IR in the range of fire. The total cost of these 8 sensors is about 36 US dollars [15].

- 1 SBC-PT single computer board. Equivalent real device available in the market is Raspberry-pi which costs 67 US dollars.
The total cost of the proposed prototype is only $70 \mathrm{US}$ dollars which is very cheap comparative to any UAVs or satellites. This cost is excluding of the cost of the Raspberry-pi as it is not part of the prototype.

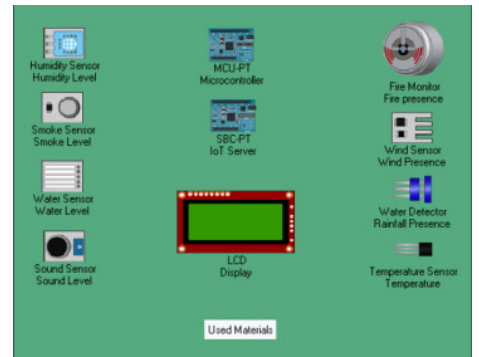

Figure 1: Materials used to build prototype and remote server.

\subsection{Methodology}

The overall system architecture as well as the workflow is depicted in Figure 2. The sensors grasp data from environment and the microcontroller collects the data from sensor and sends to the remote station. The single board computer i.e. the server in the remote station receives the data sent by the prototype. The mobile operator passes data from the prototype to the internet.

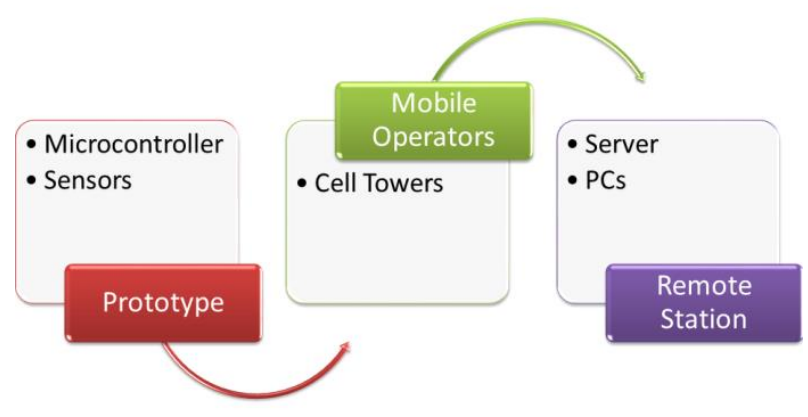

Figure 2: Overall system architecture.

The microcontroller of the prototype collects the data from the sensors connected to its ports and sends the data over a cellular network by using the wireless module PT-IOT-NM-3G/G attached to it (see Figure 3).

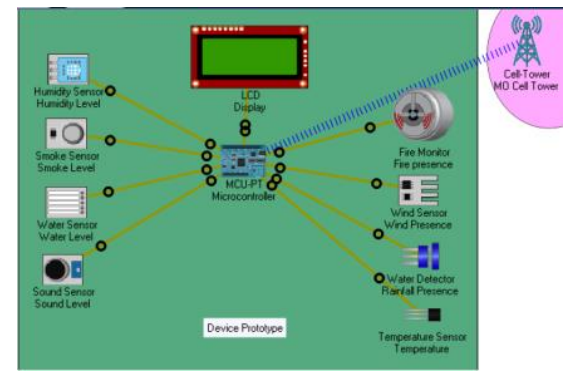

Figure 3: Prototype - first part of system architecture.

Wired broadband network infrastructure is not available in the Sundarbans area. One of the cellular phone operators named Teletalk provides network coverage in the forest area. Therefore, the cellular network is used to act 
as the physical media for transmitting data from the prototype to the remote server (see Figure 4).

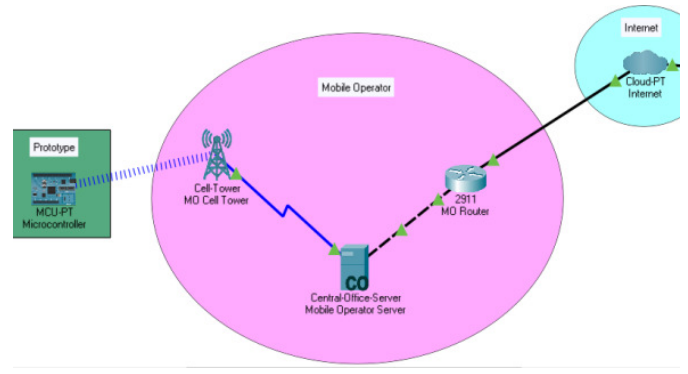

Figure 4: Mobile operator - middle part of system architecture.

A single board computer SBC-PT is used as the remote server which receives data from the distant forest, store and process the data as user requirements (see Figure 5).

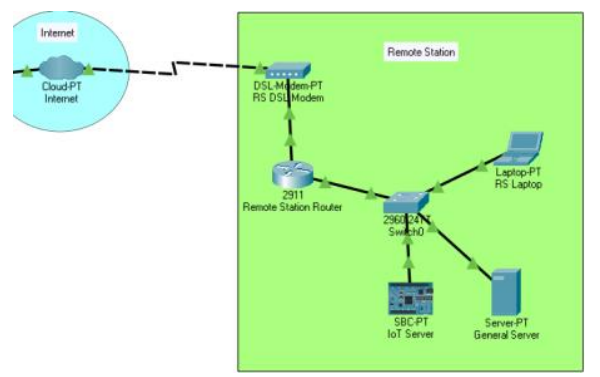

Figure 5: Remote station - last part of system architecture.

The python code that collects and sends data from the prototype is shown in Figure 6 and Figure 7. And the code which is running on the remote server SBC-PT is shown in Figure 8. The cisco packet tracer has the option to write the code in javascript as well.

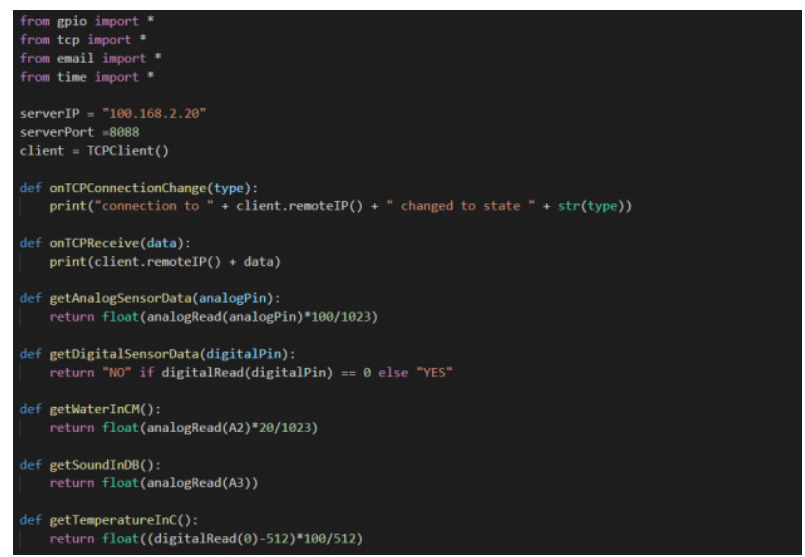

Figure 6: Source code running in the microcontroller -1 .

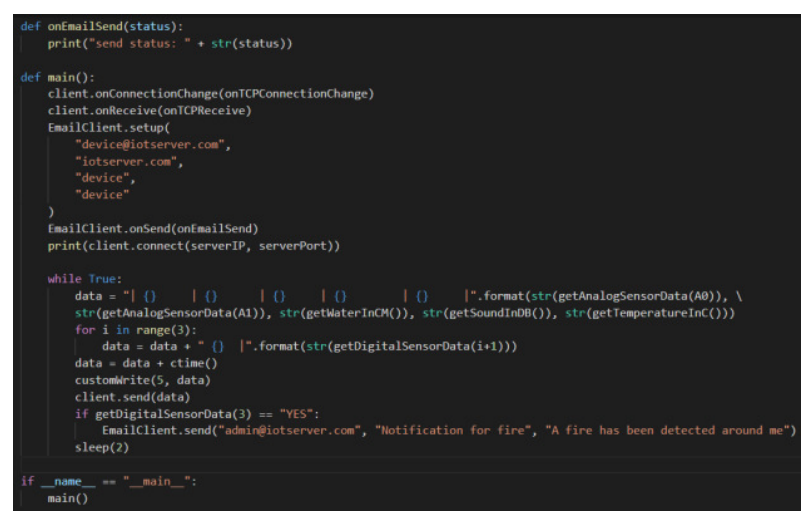

Figure 7: Source code running in microcontroller -2.

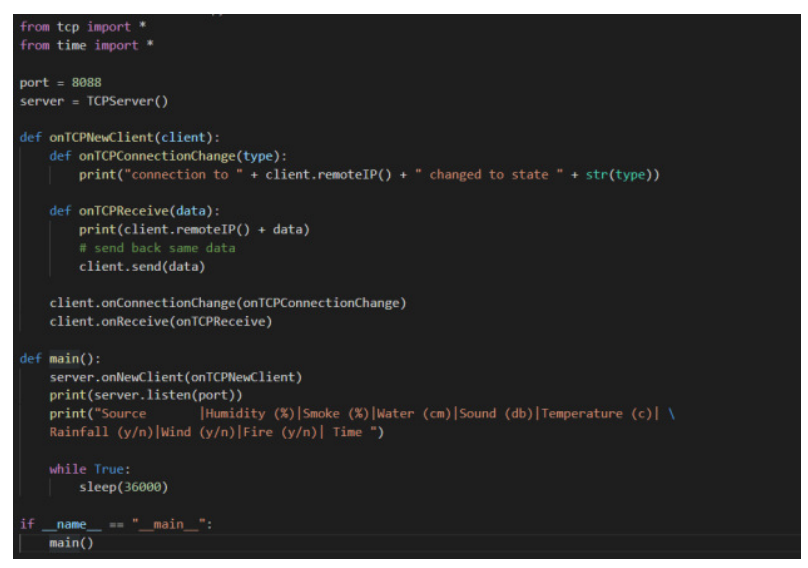

Figure 8: Source code of the remote server.

\section{Results}

The data of eight environment parameters namely humidity, smoke, water, sound, temperature, rainfall, wind and fire are shown from second column to ninth column orderly in Figure 9. The first and last columns represent source IP address and received time respectively.

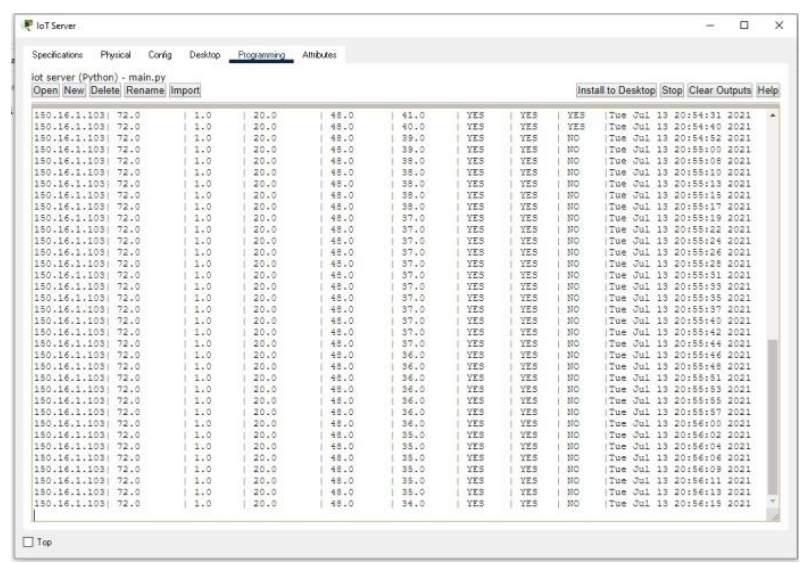

Figure 9: Environment data received in remote server.

When the prototype detects any fire, it immediately sends a notification to the administrator. A notification email sent to the remote administrator is shown in Figure 10. 


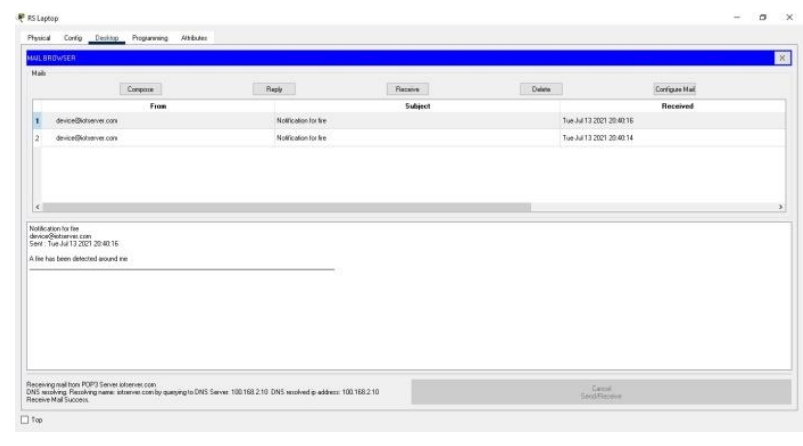

Figure 10: Email notification sent by the prototype.

\section{Discussion}

The proposed device is tested for 5 hours in the cisco packet tracer simulation tool where a test environment like mangrove forests is created using several actuators. It grasps data from the test environment and transmits to the remote server using TCP/IP continuously in 2 seconds interval. For the sake of simplicity custom socket is used instead of MQTT for transmitting data. The real implementation should use MQTT protocol as it is more lightweight, efficient, reliable and secure for IoT than TCP/IP. The readings captured in the remote server are updated in every 2 seconds. However, in the case of real implementation the prototype will be used to send data on a hourly or even daily basis depending on the requirements. The data received in the server proves the effectiveness of the prototype. Instead of this private server any cloud server can be used to receieve, store and process these data. The prototype also sends an alert message to the remote administrator using SMTP in any emergency situations such as fire detection. The proper functionality of the prototype proves the feasibility of it for low cost (see Table 1) remote monitoring of the mangrove forests of Sundarbans.

Table 1: Cost of the prototype

\begin{tabular}{|c|c|}
\hline Item & Cost \\
\hline Arduino Uno & $\$ 16$ \\
\hline Wireless module & $\$ 18$ \\
\hline Sensors & $\$ 36$ \\
\hline Total & $\$ 70$ \\
\hline
\end{tabular}

\section{Conclusions}

A prototype of an IoT device for monitoring the world's largest mangrove forests of Sundarbans is designed, constructed and examined in a simulated environment in this research. The experiment shows that the proposed device is feasible to remotely monitor the forest properly. This study also concludes that the prototype is very cheap as well because it costs only 70 US dollars (see Table 1).

The functionality of the device can be extended by incorporating more advanced sensors to collect data such as gas concentration in the air, soil nutrients and so on. Solar panels can be added to the device for providing powers.

\section{References}

[1] C. Giri, Recent Advancement in Mangrove Forests Mapping and Monitoring of the World Using Earth Observation Satellite Data, Remote Sensing 13(4). (2021) 563, https://doi.org/10.3390/rs13040563.

[2] Y. Jiang, L. Zhang, M. Yan, J. Qi, T. Fu, S. Fan, B. Chen, High-Resolution Mangrove Forests Classification with Machine Learning Using Worldview and UAV Hyperspectral Data, Remote Sensing 13(8) (2021) 1529.

[3] K. Mirakhorlou, S. Teimouri, M. Abadeh, Mapping potential of mangrove forests based on site demands (Geomorphological factors and physico-chemical characteristics of soil and water), Environ. Conserv 23 (2017) 90-97.

[4] M. Ruwaimana, B. Satyanarayana, V. Otero, A. M. Muslim, M. A. Syafiq, S. Ibrahim, D. Raymaekers, N. Koedam, F. Dahdouh-Guebas, The advantages of using drones over space-borne imagery in the mapping of mangrove forests, PloS one 13(7) (2018) e0200288.

[5] T. D. Pham, N. Yokoya, D. T. Bui, K. Yoshino, D. A. Friess, Remote sensing approaches for monitoring mangrove species, structure, and biomass: Opportunities and challenges, Remote Sensing 11(3) (2019) 230.

[6] K. D. Purkayastha, R. K. Mishra, A. Shil, and S. N. Pradhan, IoT Based Design of Air Quality Monitoring System Web Server for Android Platform, Wireless Personal Communications 118(4) (2021) 2921-2940.

[7] M. Anachkova, S. Domazetovska, Z. Petreski, V. Gavriloski, Design of low-cost wireless noise monitoring sensor unit based on IoT concept, Journal of Vibroengineering 23(4) (2021)

[8] F. Akhter, H. R. Siddiquei, M. E. E. Alahi, K. Jayasundera, S. C. Mukhopadhyay, An IoT-enabled Portable Water Quality Monitoring System with MWCNT/PDMS Multifunctional Sensor for Agricultural Applications, IEEE Internet of Things Journal (2021).

[9] P. Sumathi, R. Subramanian, V. V. Karthikeyan, S. Karthik, Soil monitoring and evaluation system using EDL-ASQE: Enhanced deep learning model for IoI smart agriculture network, International Journal of Communication Systems (2021) e4859.

[10] M. S. Uddin, E. R. V. Steveninck, M. Stuip, M. A. R. Shah, Economic valuation of provisioning and cultural services of a protected mangrove ecosystem: A case study on Sundarbans Reserve Forest, Bangladesh, Ecosystem Services 5 (2013) 88-93.

[11] A. N. M. Abdullah, N. Stacey, S. T. Garnett, B. Myers, Economic dependence on mangrove forest resources for livelihoods in the Sundarbans, Bangladesh, Forest Policy and Economics 64 (2016) 15-24.

[12] A. Jesin, Packet Tracer Network Simulator, Packt Publishing Ltd, 2014.

[13] Arduino UNO R3 board with DIP ATmega328P, https://www.walmart.com/ip/Arduino-UNO-R3-boardwith-DIP-ATmega328P/133534784, [09.08.2021].

[14] SIM808 Module GSM GPRS GPS Development Board IPX SMA with GPS Antenna for Arduino Raspberry Pi Support $2 \mathrm{G} \quad 3 \mathrm{G} \quad 4 \mathrm{G} \quad$ SIM Card, https://www.aliexpress.com/item/1005001967026161.ht 


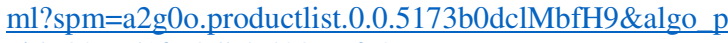
vid $=807751 \mathrm{fc}-8 \mathrm{db} 4-4387-\mathrm{af} 70$ -

84777f58bd1c\&algo_exp_id=807751fc-8db4-4387-af7084777f58bd1c-2, [09.08.2021].
[15] KOOKYE 16 in 1 Smart Home Sensor Modules Kit for Arduino Raspberry Pi DIY Professional, https://www.walmart.com/ip/KOOKYE-16-in-1-SmartHome-Sensor-Modules-Kit-for-Arduino-Raspberry-PiDIY-Professional/392581400, [09.08.2021]. 